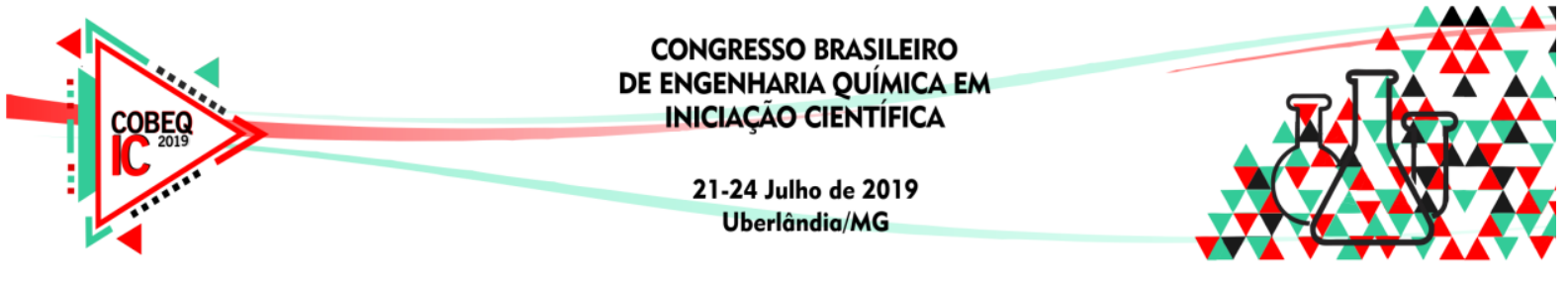

\title{
EFEITOS DA UMIDADE SOBRE AS CARACTERÍSTICAS FÍSICAS DE GRÃOS DE MUCUNA (Mucuna pruriens) E AS PROPRIEDADES BULK DO LEITO FIXO
}

\author{
A. A. F. CÂNDIDO ${ }^{1}$, I. C. D. PESSOA ${ }^{1}$ e R. N. CUNHA ${ }^{1}$ \\ ${ }^{1}$ Centro Universitário de Patos de Minas, Departamento de Engenharia Química \\ E-mail para contato: isabellacdp@unipam.edu.br
}

\begin{abstract}
RESUMO - A espécie Mucuna pruriens (L.) Dc., típica de regiões tropicais, é conhecida por suas propriedades medicinais, devido à presença da levodopa, fármaco utilizado no tratamento de Parkinson. A secagem de sementes in natura se faz necessária para prolongar a sua vida útil. Dentre os secadores, o leito fixo vem sendo amplamente utilizado para o estudo dos fenômenos envolvidos no processo de secagem. Entretanto, características físicas das partículas e propriedades bulk do leito empacotado exercem influência sobre os fenômenos de transferência de calor e massa. O presente estudo tem como objetivo avaliar os efeitos da umidade de sementes de mucuna sobre as características físicas das partículas e do leito fixo. Para tanto, as sementes foram umidificadas com água destilada e determinadas as propriedades (dimensões lineares, área superficial, volume, esfericidade, circularidade e massa específica). Para o leito fixo foram definidos os parâmetros massa específica bulk e porosidade. Os resultados apontam que a umidade da semente exerceu efeitos estatisticamente significativos sobre as propriedades morfométricas bem como o empacotamento do leito.
\end{abstract}

\section{INTRODUÇÃO}

A Mucuna pruriens, leguminosa nativa da Índia e Antilhas, é típica de regiões úmidas e tropicais, sendo comumente utilizada como adubação verde. A Adubação verde é uma prática agrícola de rotação de culturas, cujo objetivo é melhorar a capacidade produtiva do solo. A mucuna possui capacidade nutricional semelhante à soja, com significativos teores de proteínas, lipídeos, minerais e outros nutrientes.

As sementes de mucuna são utilizadas como alimento em diversos países, entretanto, seu uso é limitado devido à presença de substâncias como alguns compostos fenólicos, lectinas, inibidores de proteases, fitatos e saponinas, as quais podem interferir na digestão e absorção de nutrientes (Siddhuraju e Becker, 2005). Sendo assim, seu consumo deve ser precedido por tratamento térmico prévio. O preparo doméstico devido à desnaturação térmica reduz as concentrações das substâncias antinutricionais citadas, mantendo-as em valores aceitáveis para o consumo (Siddhuraju e Becker, 2001).

Estudos científicos associam o uso da semente de mucuna ao tratamento da doença de Parkinson, devido ao isolamento da levodopa (L-Dopa), fármaco desenvolvido a partir dessa substância quiral (Manyam, 1990; Cassani et al., 2016). 


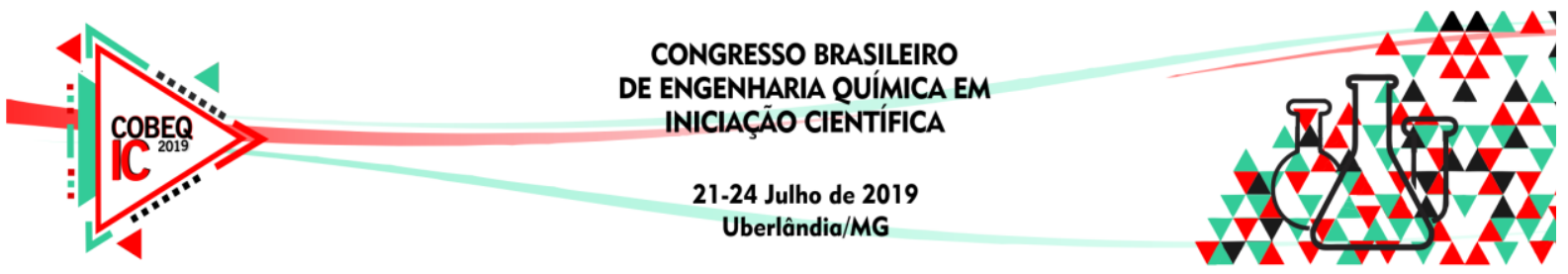

Ressalta-se que as sementes possuem significativos teores de umidade, sendo necessário efetuar a redução desta umidade, de modo a prolongar sua vida útil. A secagem, operação unitária destinada à retirada de água de um sólido por meio de energia térmica, contribui para o armazenamento e evita reações de deterioração que possivelmente venham a ocorrer (Faria, 1998).

Um dos sistemas mais empregados na indústria, o leito fixo, contém uma quantidade estacionária de sólidos pela qual perpassa o ar quente, o qual troca calor com o produto, retirando a umidade indesejada (Jorge et al., 2004). As propriedades bulk de leitos fixos e as características físicas das partículas empacotadas influenciam de forma significativa sobre a fluidodinâmica do leito, bem como nos fenômenos de transferência de calor e massa envolvidos na secagem. Nesse contexto, o presente estudo tem como propósito avaliar os efeitos da umidade das sementes de mucuna frente às características físicas das partículas e as propriedades bulk do leito fixo.

\section{MATERIAIS E MÉTODOS}

\subsection{As sementes de mucuna}

As sementes de mucuna pertencentes a cultivar mucuna cinza, utilizadas como objeto de estudo, foram adquiridas na empresa BRseeds situada em Araçatuba-SP. Os experimentos para caracterização física das sementes e do leito fixo foram conduzidos no Laboratório de Engenharia Química do Centro Universitário de Patos de Minas-UNIPAM. As sementes foram reumidificadas artificialmente por meio de borrifamentos com água destilada, mantendo-as refrigeradas por cerca de 3 a 5 dias. A determinação da umidade foi realizada por método gravimétrico utilizando estufa a $105^{\circ} \mathrm{C}$.

\subsection{As propriedades físicas}

\subsubsection{Dimensões lineares}

A determinação das dimensões características das partículas foi realizada por meio de medições utilizando o paquímetro digital com precisão de 0,01 , sendo determinados os diâmetros mutuamente perpendiculares $\left(\mathrm{a}, \mathrm{b}\right.$ e c). $\mathrm{O}$ diâmetro geométrico das partículas $\left(\mathrm{D}_{\mathrm{g}}\right)$ foi calculado pela Equação (1). Foram mensuradas de modo aleatório cerca de 50 sementes.

$$
D_{g}=(a \cdot b \cdot c)^{1 / 3}
$$

Admitindo-se que a semente de mucuna possui formato de esferoide triaxial (Figura 1), a área superficial $\left(A_{p}\right)$ e o volume $\left(V_{p}\right)$ foram calculados pelas Equações (2) e (3), respectivamente.

$$
\begin{aligned}
& A_{p}=\pi(a \cdot b \cdot c)^{2 / 3} \\
& V_{p}=\frac{\pi a \cdot b \cdot c}{6}
\end{aligned}
$$




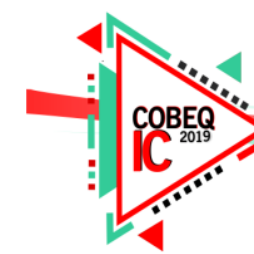

Figura 1 - Ilustração das dimensões características da semente de mucuna, considerando esferoide triaxial (a) comprimento; (b) largura e (c) espessura.
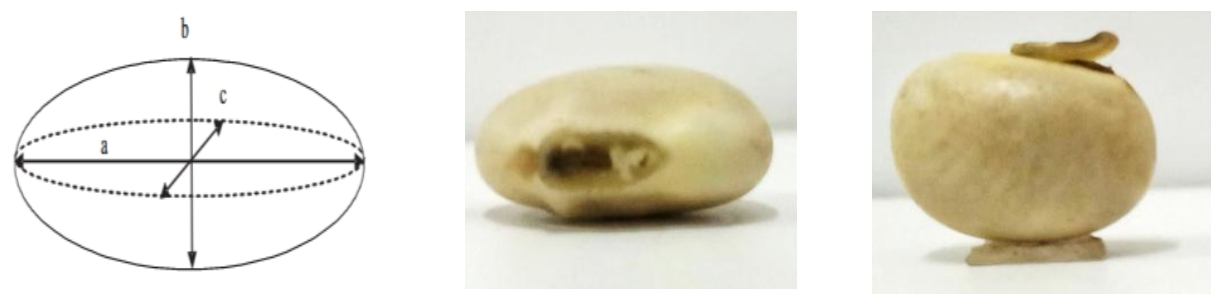

\subsubsection{Circularidade de Esfericidade}

A circularidade e a esfericidade foram determinadas pelas Equações 4 e 5, respectivamente (Mohsenin, 1970).

$$
\begin{gathered}
C_{p}=\frac{b}{a} \cdot 100 \\
\varnothing=\frac{\sqrt[3]{a \cdot b \cdot c}}{a}
\end{gathered}
$$

\subsubsection{Massa específica aparente}

A massa específica aparente considera o volume total da amostra, inclusive o espaço vazio entre os grãos que a compõem. $\mathrm{O}$ método utilizado foi a picnometria sendo a massa específica aparente calculada pela Equação 6, em que $V_{t}$ é o volume aparente ocupado pelas partículas, ou seja, o volume das partículas mais volume dos interstícios entre as partículas e $M_{p}$ a massa de partículas.

$$
\rho_{a}=\frac{M_{p}}{V_{t}}
$$

\subsubsection{Massa específica unitária}

A massa específica das sementes de mucuna foi determinada pesando-as cuidadosamente e, em seguida, determinando-se os volumes por meio das dimensões lineares (Equação 3).

\subsection{Propriedades Bulk de Leitos Fixos}

\subsubsection{Densidade bulk}

A densidade bulk $\left(\rho_{\mathrm{b}}\right)$ pode ser descrita como a razão entra a massa do meio poroso $\left(M_{L}\right)$ e o volume do leito $\left(V_{L}\right)$ empacotado, como mostrado na Equação (7). Segundo Zotin (1985) existem diferentes formas de se realizar o empacotamento do leito. Para se avaliar a densidade bulk efetuou-se o empacotamento, utilizando-se um recipiente cilíndrico graduado por derramamento de quantidade constante de material de uma mesma altura coma auxílio de um funil, seguido por batidas do recipiente contra uma superfície plana e observação do 


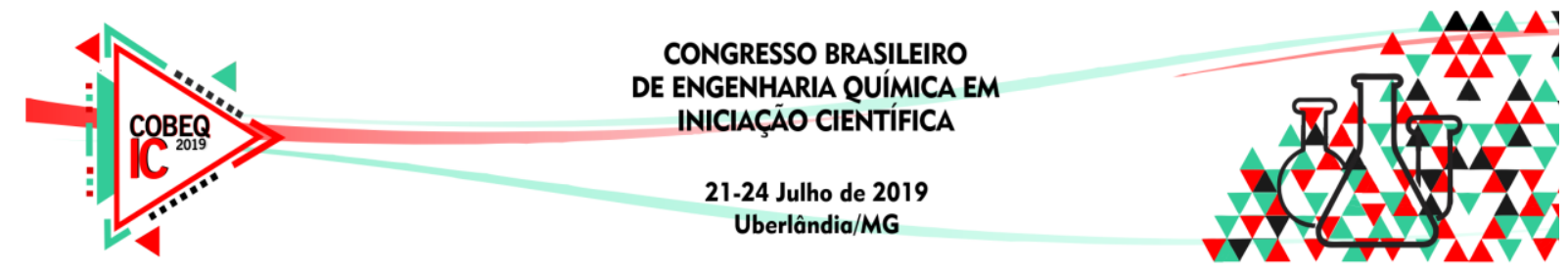

volume de material na proveta até que o mesmo seja constante. Sendo a densidade bulk determinada pela Equação 7.

$$
\rho_{b}=\frac{M_{L}}{V_{L}}
$$

\subsubsection{Porosidade bulk}

Porosidade bulk dada pela relação entre o volume de vazios de ar interpartícula e o volume total do leito, foi determinada pela relação entre as densidades, conforme Equação 8.

$$
\varepsilon_{b}=\frac{\text { Volume de vazios }}{\text { Volume total }}=1-\frac{\rho_{b}}{\rho_{r}}
$$

\subsection{Análise estatística}

A influência da umidade sobre as propriedades e parâmetros ajustados foi avaliada pela Análise de Variância (ANOVA) $(\mathrm{p} \leq 0,05)$ e teste de Tukey. As análises foram realizadas no software Statistica ${ }^{\circledR} 7.0$.

\section{RESULTADOS E DISCUSSÕES}

O conhecimento de propriedades físicas das sementes que definem a permeabilidade do leito é de fundamental importância para a compreensão dos fenômenos fluidodinâmicos e de transferência de calor e massa envolvidos na secagem em leito fixo. Na Tabela 1 são explicitados os resultados dos ensaios físicos realizados com as sementes de mucuna contendo umidades distintas. Aos valores são adicionados os desvios padrões, parâmetros estatísticos indicativos da precisão dos ensaios experimentais, e as análises estatísticas do teste de ANOVA (Tukey).

Verifica-se que o acréscimo da umidade acarretou efeitos estatísticos significativos para quase todas as dimensões lineares médias e para o diâmetro geométrico das sementes de mucuna. Resultados similares foram obtidos por Deshpande et al. (1993) e Araújo et al. (2014) na avaliação de soja e amendoim, respectivamente, os quais obtiveram dependência linear entre as dimensões e os conteúdos de umidade. Altino (2018) acrescenta que o aumento de umidade acarretou efeitos nas dimensões lineares de partículas orgânicas soja e aveia, sendo esses efeitos mais pronunciados em lentilha e cevada.

Alterações da área superficial e do volume em função da umidade ocorreram em decorrência dos aumentos das dimensões lineares. Os parâmetros circularidade e esfericidade não sofreram efeitos estatísticos significativos com o aumento da umidade. Tanto a esfericidade como a circularidade mantiveram seus valores abaixo de $80 \%$, evidenciando a incapacidade de classificar a semente de mucuna como esférica e circular, independente do teor de água. Resultados similares foram obtidos por Araújo et al. (2014).

Confrontando-se os dados de massa específica nota-se que o acréscimo de umidade contribui para a redução da massa específica aparente. Comportamento similar foi obtido para 


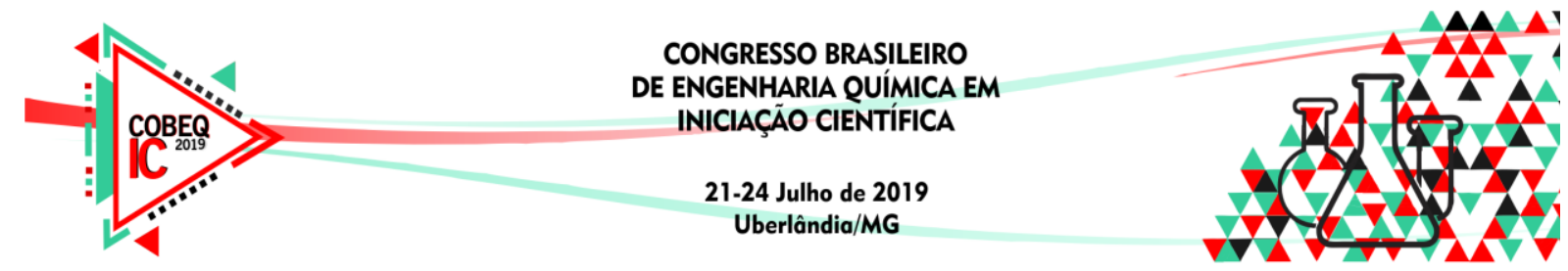

a maioria dos produtos agrícolas (Tavakoli et al., 2009). No entanto, tal fato não é evidenciado para a massa específica real, onde se observa que não existe diferença estatística significativa entre os valores.

A Tabela 02 explicita para conteúdos distintos de umidade, as magnitudes obtidas para as propriedades bulk do leito fixo, as quais representam as características físicas das partículas empacotadas.

Tabela 1- Efeitos da umidade sobre as propriedades físicas obtidas para as sementes de mucuna cinza

\begin{tabular}{|l|c|c|c|}
\hline \multirow{2}{*}{\multicolumn{1}{|c|}{ Propriedades Físicas }} & \multicolumn{3}{c|}{ Teores de água base seca } \\
\cline { 2 - 4 } & $6 \%$ & $11 \%$ & $13 \%$ \\
\hline \hline Dimensão linear a [cm] & $1,524 \pm 0,162^{\mathrm{b}}$ & $1,631 \pm 0,144^{\mathrm{a}}$ & $1,644 \pm 0,106^{\mathrm{a}}$ \\
\hline Dimensão linear b [cm] & $1,135 \pm 0,097^{\mathrm{b}}$ & $1,199 \pm 0,080^{\mathrm{a}}$ & $1,219 \pm 0,067^{\mathrm{a}}$ \\
\hline Dimensão linear c [cm] & $0,781 \pm 0,085^{\mathrm{b}}$ & $0,793 \pm 0,699^{\mathrm{ab}}$ & $0,831 \pm 0,044^{\mathrm{a}}$ \\
\hline Diâmetro geométrico [cm] & $1,104 \pm 0,094^{\mathrm{b}}$ & $1,163 \pm 0,053^{\mathrm{a}}$ & $1,185 \pm 0,058^{\mathrm{a}}$ \\
\hline Área superficial [cm $\left.{ }^{2}\right]$ & $3,854 \pm 0,650^{\mathrm{b}}$ & $4,260 \pm 0,382^{\mathrm{ab}}$ & $4,429 \pm 0,459^{\mathrm{a}}$ \\
\hline Volume [cm $\left.{ }^{3}\right]$ & $0,719 \pm 0,182^{\mathrm{b}}$ & $0,829 \pm 0,111^{\mathrm{ab}}$ & $0,885 \pm 0,138^{\mathrm{a}}$ \\
\hline Circularidade [\%] & $51,64 \pm 6,57^{\mathrm{a}}$ & $47,88 \pm 5,21^{\mathrm{a}}$ & $50,40 \pm 3,59^{\mathrm{a}}$ \\
\hline Esfericidade [\%] & $72,73 \pm 0,51^{\mathrm{a}}$ & $70,38 \pm 0,42^{\mathrm{a}}$ & $72,12 \pm 0,35^{\mathrm{a}}$ \\
\hline Massa específica aparente $\left[\mathrm{g} / \mathrm{cm}^{3}\right]$ & $0,786 \pm 0,024^{\mathrm{a}}$ & $0,743 \pm 0,022^{\mathrm{b}}$ & $0,735 \pm 0,024^{\mathrm{b}}$ \\
\hline Massa específica unitária [g/cm $\left.{ }^{3}\right]$ & $1,420 \pm 0,044^{\mathrm{a}}$ & $1,422 \pm 0,063^{\mathrm{a}}$ & $1,397 \pm 0,061^{\mathrm{a}}$ \\
\hline
\end{tabular}

Médias seguidas da mesma letra na coluna não diferem estatisticamente entre si. Foi aplicado o teste de Tukey em nível de $5 \%$ de probabilidade

Tabela 02: Efeitos da umidade sobre as propriedades bulk do leito fixo

\begin{tabular}{|l|c|c|c|}
\hline \multirow{2}{*}{ Propriedades bulk do leito } & \multicolumn{3}{|c|}{ Teores de água base seca } \\
\cline { 2 - 4 } & $6 \%$ & $11 \%$ & $13 \%$ \\
\hline \hline Densidade bulk & $0,804 \pm 0,007^{\mathrm{a}}$ & $0,746 \pm 0,006^{\mathrm{b}}$ & $0,720 \pm 0,018^{\mathrm{c}}$ \\
\hline Porosidade do leito fixo & $0,393 \pm 0,009$ & $0,416 \pm 0,007$ & $0,440 \pm 0,023$ \\
\hline
\end{tabular}

Infere-se que a densidade bulk sofreu reduções com os acréscimos dos teores de água. Resultados similares foram obtidos por Pagano e Crozza (2000) em estudo com sementes de milho e Altino (2019). No entanto, efeito inverso foi obtido para a porosidade do leito. Vale ressaltar que as variações na resistência ao transporte de calor e massa na secagem se devem a diversos fatores, como: características físicas das partículas, forma de ligação entre a estrutura do material e a água e propriedades bulk dos leitos.

\section{CONCLUSÃO}

A partir dos resultados obtidos conclui-se que variações do teor de água presente em sementes de mucuna: (i) acarretaram mudanças nas características físicas das partículas, aumentando seu diâmetro médio, sua área superficial e seu volume; (ii) não alteraram a circularidade e nem a esfericidade e (iv) contribuíram por alterações das propriedades bulk do leito fixo. 


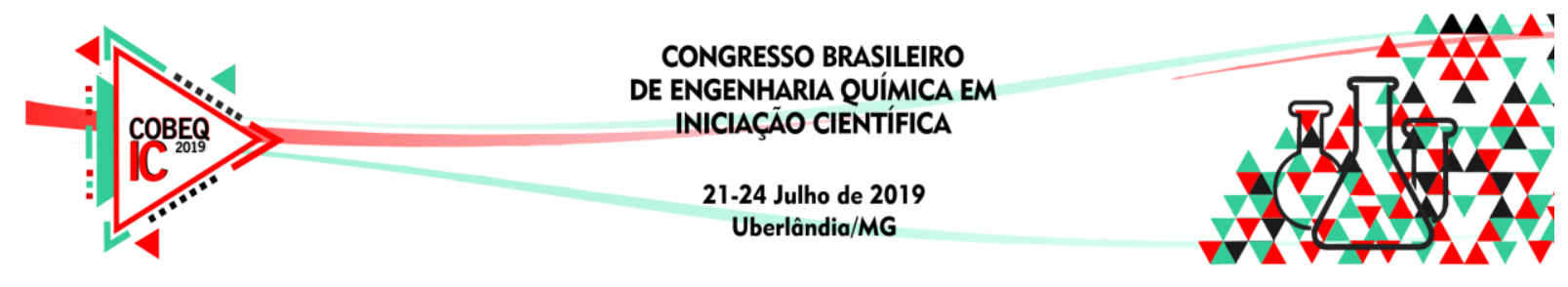

\section{REFERÊNCIAS}

ALTINO, H. O. N. Secagem em leito fixo de materiais granulares com diferentes esfericidades. 2019. 87 f. Dissertação (Mestrado) - Curso de Programa de Pósgraduação em Engenharia Química, Centro de Ciências Exatas e Tecnologia, Universidade Federal de São Carlos, São Carlos, 2019.

ARAUJO, W. D.; GONELI, A. L. D.; SOUZA, C. M. A.; GONÇALVES, A. A.; VILHASANTI, H. C. B. Propriedades físicas dos grãos de amendoim durante a secagem. Revista Brasileira de Engenharia Agrícola e Ambiental, [s.1.], v. 18, n. 3, p.279-286, mar. 2014.

CASSANI, E.; CILIA, R.; LAGUNA, J.; BARICHELLA, M.; COTIN, M.; CEREDA, E.; ISAIAS, I. U.; SPARVOLI, F.; AKPALU, A.; BUDU, K. O.; SCARPA, M. T.; PEZZOLI, G. Mucuna pruriens for Parkinson's disease: Low-cost preparation method, laboratory measures and pharmacokinetics profile. Journal Of The Neurological Sciences, [s.1.], v. 365, p.175-180, jun. 2016.

DESHPANDE, S. D.; BAL, S.; OJHA, T. P. "Physical properties of soybeans", J. Agric. Eng. Res., 56, pp.89-98, 1993.

FARIA, L. J. G. Análise experimental do processo de secagem de urucum (Bixa Orellana L.) em leito fixo. 1998. Tese de Doutorado (Engenharia Química), Campinas - SP, 1998.

JORGE, L. M. M.; FREGOLENTE, L. V.; SANTOS, O. A. A. Estimativa das propriedades térmicas efetivas de grãos em um secador de leito fixo. Ciênc. Tecnol. Aliment., Campinas - SP; v. 24, n. 2, p. 270-276, 2004.

MANYAM, B. V. Paralysis agitans and levodopa in "Ayrveda": Ancient Indian medical treatise. Movement Disorders, [s.1], v. 5, n. 1, p. 47-48, 1990.

MOHSENIN, N. Physical properties of plant and animal materials. New York: Gordon and Breach, 1970.

PAGANO, A. M.; CROZZA, D. E. "Prediction of bulk density and bed porosity from data of bed depth and moisture content for two oilseeds", Anais do XIII Congresso Brasileiro de Engenharia Química, Águas de São Pedro, SP, 2000.

SIDDHURAJU, P.; BECKER, K. Nutrition al and antinutritional composition, in vitro amino acid availability, starch digestibility and predicted glycemic index of differentially processed mucuna beans (Mucuna pruriens var. utilis): an under-utilised legume. Food Chemistry, Barking, v. 91, p. 275-286, 2005.

SIDDHURAJU, Perumal; BECKER, Klaus. Preliminary nutritional evaluation of Mucuna seed meal (Mucuna pruriens var. utilis) in common carp (Cyprinus carpio L.): an assessment by growth performance and feed utilisation. Aquaculture, [s.1.], v. 196, n. 12, p.105-123, maio 2001.

TAVAKOLI, M.; TAVAKOLI, H.; RAJABIPOUR, A.; AHMADI, H.; GHARIBZAHEDI, S. M. T. Moisture-dependent physical properties of barley grains. International Journal of Agricultural and Biological Engineering, v. 4, p. 84-91, 2009.

ZOTIN, F. M. Z. O efeito de parede em colunas de recheio. 1985. 85 f. Universidade Federal de São Carlos, 1985. 